\title{
De los Objetivos del Milenio a los Objetivos del Desarrollo Sostenible: la integración de países en vía de desarrollo por medio de la intensificación del uso de las tecnologías
}

\author{
Victoria Valdivia* \\ Raimundo González Aninat ${ }^{* *}$
}

\section{RESUMEN}

Los Objetivos del Milenio marcaron una pauta de evolución para el desarrollo de aquellos Estados en condición de vulnerabilidad, a fin de acortar la brecha de desigualdad y potenciar los factores de desarrollo humano a nivel de sociedad global. Hoy en día vemos que dicha brecha se ha profundizado entre los países desarrollados y los en vía de desarrollo, debido al impulso de la tecnología de información como nuevo factor productivo, tornándose a su vez en uno de los factores críticos para poder superar la desigualdad y dotar a los Estados subdesarrollados de mejores capacidades en el orden internacional.

\footnotetext{
* Magíster en Estudios Internacionales. Investigadora asociada al Instituto de Estudios Avanzados de la Universidad de Santiago, Chile. [victoriaf.valdiviac@gmail.com].

** Embajador en Misión Especial y asesor de la Cancillería. Profesor de la Facultad de Derecho de la Universidad de Chile y profesor visitante de la Flacso. Miembro del Panel de expertos legales en Derecho Espacial de la Corte Permanente de Arbitraje de La Haya. [rgonzalezaninat@gmail.com].
}

Recibido: 15 de julio de 2016/ Modificado: 30 de septiembre de 2016/ Aceptado: 6 de noviembre de 2016

Para citar este artículo

Valdivia, V. y González Aninat, R. (2017). De los Objetivos del Milenio a los Objetivos del Desarrollo Sostenible: la integración de países en vía de desarrollo por medio de la intensificación del uso de las tecnologías. OAsIs, 25, 149-157.

DOI: https://doi.org/10.18601/16577558.n25.09 
Palabras clave: integración latinoamericana, desarrollo espacial, tecnología, desarrollo.

\section{From the Millennium}

\section{Development Goals to the} Sustainable Development Goals: The integration of developing countries through the intensification of the use of technologies

\section{ABSTRACT}

The Millennium Development Goals marked a pattern in the evolution of the development of states in a condition of vulnerability in order to bridge the gap of inequality and promote human development factors at the level of global society. Today, we see that the gap between developed and developing countries has widened due to the promotion of information technology as a new production factor, becoming in turn one of the critical factors to overcome inequality and to endow developing states with better capabilities in the international order.

Key words: Latin American integration, spatial development, technology development.

\section{INTRODUCCIÓN}

Los Objetivos del Milenio de las Naciones Unidas, con enfoque hacia los países en vías de desarrollo, han marcado el desenvolvimiento de la agenda pública y política internacional, con el fin de afrontar las principales problemáticas de dichos países, tales como el hambre, la pobreza estructural, los conflictos armados internos y transfronterizos, entre otros, todos síntomas del aumento de la brecha de desigualdad e inequidad que enfrentan estos actores.

Sin embargo, acorde al modelo de producción que rige la actividad económica mundial, alcanzar el desarrollo económico involucra una continua y sostenida degradación del medio ambiente como causa de la intensificación de la actividad industrial, situación que se complejiza toda vez que los países en vías de desarrollo se ubican geográ-

FIGURA 1. RESERVAS DE AGUA DULCE EN EL MUNDO, POR REGIÓN

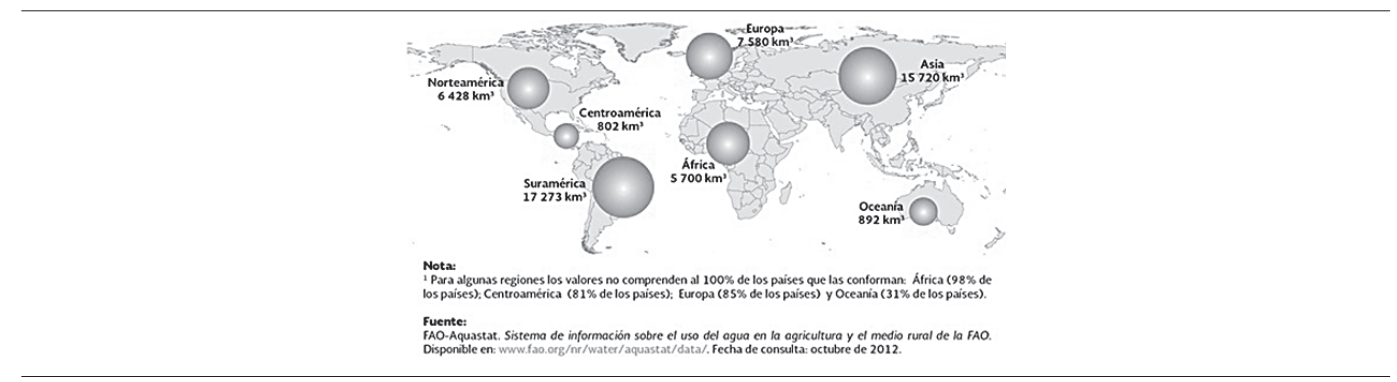

Fuente: FAO Aquastat (2012). 
ficamente en los mayores nichos de recursos estratégicos, como por ejemplo el recurso del agua, tal como se muestra en la figura 1.

Los Objetivos del Milenio han servido como diagnóstico en la lucha contra la desigualdad, y cuando menos han puesto el punto de interés mundial en aquellos factores que profundizan relaciones dispares en las relaciones internacionales. En la práctica responden al orden internacional sustentado en las capacidades de cada sujeto, y estas reposan en factores asociados a la acumulación de riqueza, concentrada esta en aquellos que han tenido un desarrollo industrial más profundo, los cuales controlan factores productivos como consecuencia de un desarrollo histórico sostenido, el cual con todo, ha provocado la degradación medioambiental.

La intensificación de la actividad industrial es necesaria para poder dotar a los países en vías de desarrollo y a su población de capacidades más equitativas a fin de lograr en la prontitud del tiempo el anhelado desarrollo, elevando los indicadores de desarrollo humano y reduciendo el surgimiento de desafíos globales como consecuencia de la inequidad y pobreza estructural, tales como las actividades de narcotráfico, tráfico de armas y otros crímenes asociados a la necesidad de incrementar capacidades económicas.

En este nuevo desafío de encontrar una alternativa que permita el desarrollo económico y la preservación del medioambiente es que en el año 2014 Naciones Unidas propone la agenda de los Objetivos del Desarrollo Sostenible (ODS).
A diferencia de los Objetivos del Milenio, que se dirigían a los países subdesarrollados, los oDs son de aplicación universal, a todos los países y a todos los niveles, y abarcan temáticas tan importantes como la lucha contra el hambre, la pobreza, la protección del medio ambiente, el bienestar o el desarrollo económico, ámbitos que afectan tanto la política interna como la acción exterior del Gobierno.

Los ods son el resultado de un proceso de negociación que se ha estado llevando a cabo en los últimos tres años, el cual ha sido valorado con una participación sin precedentes de la sociedad civil y de las partes interesadas. Cataluña, a través de la Red de Gobiernos Regionales para el Desarrollo Sostenible (nrg4sD), miembro organizador del grupo de interés oficial de autoridades locales, y de la Organización Mundial de Regiones (ORU-FOGAR), ha contribuido especialmente a que esta agenda reconozca el papel de los gobiernos regionales y locales en las políticas relacionadas con el desarrollo sostenible (La Otra Opinión, 2015).

Así pues, los 17 ods que abarcan la multisectorialidad de la actividad humana cuentan con metas y agendas para su materialización en un horizonte de 15 ańos. Meta que es posible, en la perspectiva de la inclusión de la tecnología.

En virtud de lo anterior, en este artículo se plantea la hipótesis de que la integración latinoamericana, como factor transversal de superación del subdesarrollo, es posible desde la integración en la construcción de una institucionalidad orientada hacia la industria espacial. A los fines de la hipótesis planteada se establece como objetivo general describir la relación entre crecimiento económico, desigualdad e incremento de tecnología.

Como objetivos específicos se plantea: 
- Describir los Objetivos del Desarrollo Sustentable como marco para el desarrollo cooperativo de tecnología.

- Describir las condiciones actuales del orden internacional y el desfase en los modos de producción entre sectores del sistema internacional.

- Proponer la cooperación inter e intrarregional como factor estratégico para la consolidación de la industria espacial.

\section{EL ROL DE LA TECNOLOGÍA EN UN TIEMPO DE GLOBALIZACIÓN}

El fenómeno de la globalización, entendido como la intensificación de los nodos de comunicación interactorales, trajo como gran novedad a partir de la década de los noventa el incremento de la comunicación y una creciente dependencia para la realización de actividades de cooperación entre actores tanto a niveles estatales como privados. Ello generó un escenario nunca antes visto: la inclusión de la sociedad civil en la actividad otrora reservada para los Estados. De hecho, esto ha traído consigo la horizontalización de las relaciones internacionales, ya que la interconectividad que esta genera ha permitido el advenimiento de nuevos temas y nuevos actores.

El surgimiento de Internet y la creciente conectividad de usuarios civiles a nivel global, tiene como principal producto el acortamiento de distancias. Sin importar en qué lugar del mundo se esté, por medio de las herramientas del ciberespacio (Internet) se accede de forma inmediata a información que permite la toma de decisiones. La vorágine de avance científicotecnológico ha incrementado la dependencia entre la toma de decisiones efectiva con el acceso a la información. Una una oportunidad única en un momento único.

El ciberespacio, en principio, supone acceso libre y gratuito para toda la humanidad, sin importar el origen étnico, la condición socioeconómica, la religión u otros factores de desigualdad. Es una herramienta libre, disponible por medio de ingenios que, conforme se masifican, siguiendo la lógica del mercado, tienden a bajar sus costos. Según los datos aportados por la Unión Internacional de Telecomunicaciones (ITU), en 2015 habría "1,91 mil millones de usuarios móviles en todo el mundo. Y para el 2016 la cifra se acercaría a 2,16 mil millones de usuarios. En el 2018, el panorama será aún más alentador, pues un tercio de los consumidores (o 2,56 mil millones de personas) contarán con un smartphone" (Clases de periodismo, 2014) (figura 2).

Teniendo en cuenta lo anterior, es válido preguntarse ipor qué no se ha reducido la brecha de la desigualdad si se ha observado un incremento en el acceso de usuarios al ciberespacio y, por consiguiente, existe un presunto mayor acceso a la información? Lo anterior se responde porque la conexión a dicho espacio no es gratuita. Los valores de acceso a la conexión al ciberespacio se verán incrementados en aquellos Estados que no posean autonomía en el acceso al mismo; vale decir, que no posean el desarrollo de la estructura crítica para ello: satélites de telecomunicaciones.

El desarrollo de la industria espacial permite la exploración de este escenario geográfico, resultado de la extensión de la biosfera, e incluso del sistema internacional, por medio 
FIGURA 2. PORCENTAJE DE USUARIOS DE SMARTPHONE EN EL MUNDO

Smartphone User and Penetration Worldwide, 2013-2018

billions, \% of mobile phone users and \% change

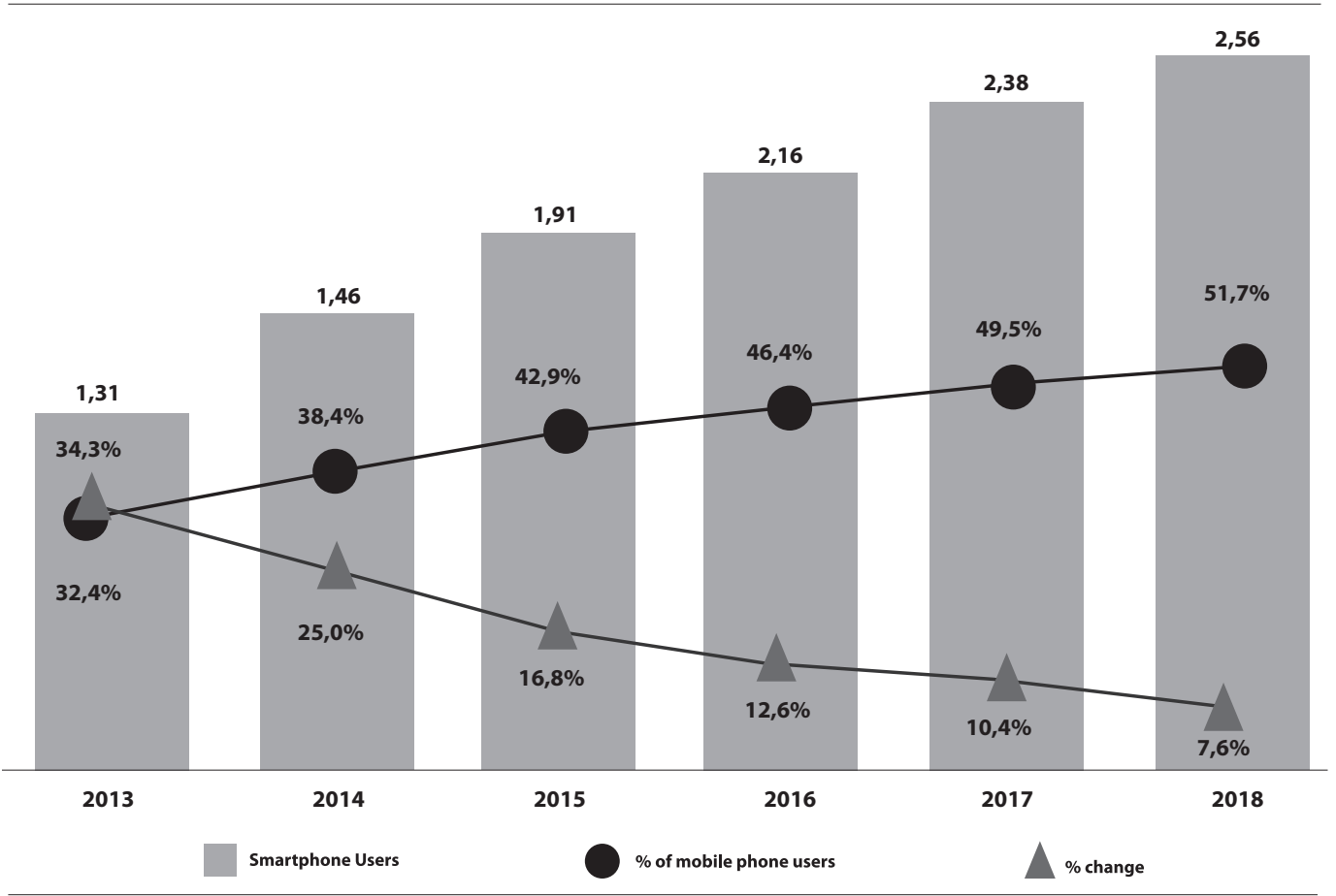

Note: individuals of ani age who own al least one smarphone and use the smarphone(s) at least once per month.

Fuente: eMarketer. Dec 2014.

del posicionamiento de ingenios en espacio ultraterrestre.

Los satélites, como estructura crítica de la información, levantan y producen datos cuyo análisis, en conjunción con el conocimiento humano, hace posibles actividades como la supervisión de los océanos, las comunicaciones, la televisión satelital, el monitoreo de fenómenos naturales, entre otros. Las más de 200 aplicaciones satelitales y la venta de estos servicios, en una lógica de mercado, permiten a los dueños de estas estructuras potenciar sus capacidades económicas. Así pues, subsistirán dos categorías de actores: productores y consumidores, siendo los productores los propietarios de un modo de producción que intensifica la relación de dependencia hacia el producto: información, y que dadas las asimetrías existentes, puede llegar a acrecentar las brechas de desigualdad. 
La creciente dependencia de los beneficios de las aplicaciones espaciales, en un contexto de desigualdad en virtud de la carencia estructural a nivel interno para producir ingenios satelitales ${ }^{1}$, aumenta la desigualdad en la oportunidad y los costos de acceso a la conexión que concede la información, la cual es construida por medio de la acción de todos los usuarios de Internet que, de una forma u otra, se transforman en factores de la cadena productiva de información sin recibir retribución por ello.

Este es un escenario difícil para los países en vías de desarrollo, puesto que la capacidad de desarrollar ingenios satelitales que permitan optimizar los costos de acceso a la conexión para obtención de información relevante para la toma de decisiones precisa de infraestructura y de la formación de capital humano avanzado; de otra forma será imposible alejarse del umbral de la extrema pobreza. ¿Cómo avanzar hacia el espacio y sus beneficios para afrontar la desigualdad de cara al futuro?

\section{Los Objetivos del Desarrollo Sostenible. OBJETIVO 17: FORTALECER LOS MEDIOS DE ejeCución y ReVitalizar la Alianza Mundial para el Desarrollo Sostenible}

La propuesta del ods 17, "Fortalecer los medios de ejecución y revitalizar la Alianza Mundial para el Desarrollo Sostenible", se transforma en una ruta de acceso a la cooperación necesaria a nivel intersectorial, dentro y entre las regiones del mundo, a fin de poder generar el desarrollo de un sector como el espacial, que sea capaz de resolver los problemas sociales que se encuentran insertos en las agendas públicas nacionales e internacionales, tales como problemas de acceso a recursos naturales vitales, a la información, a la gestión de crisis y desastres devenidos de fenómenos asociados al cambio climático, entre otros.

El oDs 17 está orientado a solucionar el problema de insumos y recursos para poder articular acciones que permitan el cumplimiento de otros oDs tales como el 9 (construir la infraestructura resiliente, promover la industrialización inclusiva y la innovación) y el osD 10 (reducir la desigualdad en y entre los países); estos vinculados directamente con el desarrollo del sector industrial espacial como insumo transversal a la gestión de demandas sociales y ventanas de oportunidad de desarrollo social. El osd 17 establece que:

Para que una agenda de desarrollo sostenible sea eficaz se necesitan alianzas entre los gobiernos, el sector privado y la sociedad civil. Estas alianzas inclusivas se construyen sobre la base de principios y valores, una visión compartida y objetivos comunes que otorgan prioridad a las personas y al planeta, y son necesarias a nivel mundial, regional, nacional y local.

\footnotetext{
1 Carencia que es multisectorial, puesto que es necesario infraestructura en materias de educación, formación de capital humano avanzado, falta de cohesión política, falta de institucionalidad, inversionistas, etc. Dichos insumos de infraestructura difícilmente son sorteables en contextos de subdesarrollo debido al surgimiento de factores con necesidad de solución inmediata como los relativos al acceso al alimento, agua, servicios básicos que garanticen la supervivencia de la sociedad nacional.
} 
Es preciso adoptar medidas urgentes encaminadas a movilizar, reorientar y aprovechar billones de dólares de recursos privados para generar transformaciones a fin de alcanzar los Objetivos de Desarrollo Sostenible. Se necesitan inversiones a largo plazo, por ejemplo, la inversión extranjera directa, en sectores fundamentales, en particular en los países en desarrollo. Entre estos sectores figuran la energía sostenible, la infraestructura y el transporte, así como las tecnologías de la información y las comunicaciones. El sector público deberá establecer una orientación clara al respecto. Deben reformularse los marcos de examen y vigilancia, los reglamentos $y$ las estructuras de incentivos que facilitan esas inversiones a fin de atraer inversiones y fortalecer el desarrollo sostenible. También deben fortalecerse los mecanismos nacionales de vigilancia, en particular las instituciones superiores de auditoría y la función de fiscalización que corresponde al poder legislativo (Naciones Unidas, 2016).

Bajo la lógica del ods 17, es necesario aumentar incentivos a la cooperación en y entre las regiones, a fin de potenciar el desarrollo de una industria que facilite la equiparación de capacidades entre actores estatales por medio del desarrollo económico sin perjudicar el medioambiente; industria ligada a la innovación y exploración de nuevas y más eficientes fuentes energéticas, tal como es la industria espacial, cuyo producto inserta a los países en el centro de la discusión de innovación y nuevos insumos para la toma de decisiones, así como la custodia, mediante actividades ligadas al monitoreo satelital, de los recursos geoestratégicos del planeta, tales como acuíferos, reservas de agua dulce, pulmones terrestres, actividad glaciar, etc.

\section{CONCLUSIONES}

Nos encontramos, como humanidad, en un momento crítico; lo más próximo a un punto de inflexión en donde los flagelos de la desigualdad pueden ser dejados como parte de una historia que esperamos no reiterar; una ventana de oportunidad para los países en vías de desarrollo, a fin de incorporarse a los desafíos del nuevo milenio, con una conciencia mucho más profunda del cuidado de nuestra casa común.

En consecuencia con lo expuesto, la inclusión de la tecnología para el desarrollo económico y social, cada vez se liga con mayor fuerza a la existencia de estructuras críticas de información: satélites. La industria espacial es la actividad que lidera los progresos científicos para hacer frente a los desafíos globales que afectan a la humanidad desde la multidimensionalidad.

Respecto a los desafíos o las amenazas no convencionales, se resalta la profunda necesidad de incentivar mecanismos de cooperación entre unidades estatales a fin de que la acción coordinada e integrada de los miembros de la gran aldea global podamos protegernos los unos a los otros, tal como establecen los oDs 13 y 14 respecto al calentamiento global y los océanos.

Es solo con la cooperación que la humanidad puede hacer frente a escenarios de desastres naturales como un terremoto y otros riesgos y crisis. Por ello, es necesario seguir promoviendo y fortaleciendo las Conferencias Espaciales de Las Américas (CEAS), de las que Chile ha sido su principal impulsor desde la década de los noventa. 
Es conocido que uno de los resultados de la desigualdad es la incapacidad autárquica de los Estados para acceder a los beneficios y desarrollos tecnológicos. Al poseer menores capacidades, raramente estas alcanzan para derivar a sectores terciarios y potenciar su desarrollo, pues se basan fuertemente en el sector primario de la economía, vale decir, en un modelo extraccionista que no potencia la innovación y el desarrollo. Frente a ello, es de carácter urgente desarrollar instancias de integración a nivel intrarregional, en donde converjan los esfuerzos multisectoriales.

Se plantea a nivel intrarregional, adaptando la teoría de la escuela inglesa de relaciones internacionales, la cual señala que una misma unidad territorial que ha pasado por procesos históricos análogos y enfrenta problemáticas de origen similar, debería tender con mayor naturalidad a la cooperación. América Latina es una buena prueba de ello; sin importar banderas o nacionalidades hay un punto en donde todos los Estados convergen en sus intereses: proteger a la población nacional. En un análisis transversal, las unidades que componen el territorio se asemejan entre sí desde factores como la heterogeneidad de su población. No existe en América Latina un origen étnico único.

La multiculturalidad, una de las mayores riquezas de nuestra América, nos permite adaptar diversas formas del saber hacer; conocimiento técnico y cultural que hace a nuestra población altamente adaptable a los distintos entornos.

Potenciar el desarrollo espacial por medio de una institucionalidad compartida, nos permitirá optimizar recursos y alcanzar los objetivos del desarrollo sostenible por medio de la aplicación de tecnología satelital, a un menor costo de producción. Asimismo, hará posible generar fórmulas para desarrollar, de consuno, la actividad industrial espacial. De este modo podremos construir un relato estratégico que supere las eventuales dificultades de la contingencia que oscurecen el logro de los ods en las áreas que resulten pertinentes.

Sinergia, cooperación e interés en común son valores guías que nos permitirán consolidar una tarea pionera para los países en vías de desarrollo. Asimismo, mediante la cooperación se intensifica la hipótesis de paz; contribuimos a un mundo más seguro, más amigable y más igualitario para las generaciones futuras.

En esta visión de futuro, donde construimos lazos profundos de cooperación para nosotros y las generaciones del mañana, es imprescindible contar con una actualización de los paradigmas del derecho internacional, a la luz de los oDs, con la promoción y el fortalecimiento de un Estado de derecho que tenga en cuenta el dinamismo de la ciencia y la tecnología para estructurar un desarrollo social inclusivo con base en la Agenda pos $2015 \mathrm{del}$ Secretario General de las Naciones Unidas.

\section{REFERENCIAS}

Arellano, J. P. (2011). El cobre como palanca de desarrollo para Chile. Recuperado de http://www.cieplan. org/media/publicaciones/archivos/227/El_Cobre_como_palanca_del_desarrollo_de_Chile.pdf Bernal-Meza, R. (2014). Fundamentos y aspectos controversiales sobre el Segundo Mundo. Jornadas Internacionales: Pensar los grandes espacios y grandes 
procesos. Santiago de Chile: Instituto de Estudios Avanzados - Academia Diplomática de Chile.

Clases de Periodismo (2016). Cantidad de Smartphones en el mundo. Recuperado de http://www.clasesdeperiodismo.com/2014/12/23/en-2016-habrados-mil-millones-de-usuarios-de-smartphonesen-el-mundo/

FAO- Aquastat (2012). Sistema de información sobre el uso del agua en la agricultura y el medio rural. Recuperado de http://www.fao.org/rur/water/ aquastat/data

Bruckmann, M. (2012). América Latina y la disputa global. En Bruckmann, M. Recursos naturales y la geopolitica de la integración sudamericana (pp. 108). Quito: IAEN.

Clases de periodismo (2014). En 2016 habrá dos mil millones de usuarios de smartphones en el mundo. Recuperado de http://www.clasesdeperiodismo. com/2014/12/23/en-2016-habra-dos-mil-millones-de-usuarios-de-smartphones-en-el-mundo/

Gobierno de la República Popular de China (2008). Texto íntegro del documento sobre la politica de China hacia América Latina y el Caribe. Recuperado de http://www.eumed.net/rev/china/09/ documento.pdf

Gómez, P. B. (2014). El ascenso económico de China: implicaciones estratégicas para la seguridad global.
Recuperado de http://pendientedemigracion. ucm.es/info/eid/pb/Bustelo\%20-\%20CESEDEN09china.pdf

Keohane, R. y Nye, J. (1977). Power and Interdependence: World politics in transition. Boston: Little Brown.

La Otra Opinión (2015). Cataluña: el Gobierno aprueba plan nacional para los OSD. Recuperado de https://dcriado.wordpress.com/2015/11/27/ cataluna-el-gobierno-aprueba-plan-nacionalpara-los-osd/

Morgenthau, H. (1992). Escritos sobre politica internacional. Madrid: Tecnos.

Naciones Unidas (2016). Objetivos para un desarrollo sostenible. Recuperado de http://www.un.org/ sustainabledevelopment/es/inequality/

Nye, J. (1990). Bound to Lead. The changing nature of american power. Oxford: Oxford University Press.

Reyes Matta, B. (2014). Nuevo diálogo político de América Latina y China. Jornadas de politica internacional: Pensando los grandes espacios y los grandes procesos. Santiago de Chile: Instituto de Estudios Avanzados - Academia Diplomática de Chile.

Rodríguez, I. y Leiva, D. (2013). El soft power en la política exterior de China: consecuencias para América Latina. Polis, 46. 
